\title{
Vision Based Object Following for Underwater Vehicles
}

\author{
by $\quad$ B. A. A. P Balasuriya* "Tamaki Ura*, Member
}

\begin{abstract}
Summary
This paper addresses the use of a vision sensor in the Underwater Vehicle (UV) feedback control loop discussing the nature of underwater images captured by CCD cameras. A prefilter to reduce unwanted features unique to underwater environments and two active vision algorithms are proposed for autonomous underwater object following. The two algorithms focus on typical underwater applications such as underwater cable tracking and underwater object following. The experimental results demonstrate that these algorithms can be implemented in real time with the use of small hardware systems.
\end{abstract}

\section{Introduction}

Current data acquisition methods for underwater vehicles such as ROVs (Remotely Operated Vehicles) and AUVs (Autonomous Underwater Vehicles) are very costly and severely limited ${ }^{2}$. Direct, long-duration exploration of the ocean by human is also limited and involve high risk even at depths of a few hundred meters.

AUVs must meet the needs of ocean exploration/ exploitation while overcoming some of the limitations of humans and ROVs. Accordingly, AUVs must be intelligent enough to take decisions in unforeseen, highly complex underwater environments. In order to achieve completely autonomous underwater systems, it is necessary to integrate many types of sensors. Although, at present, acoustic sensors are widely used in underwater applications, they encounter problems such as acoustic shading and multipath effect when close range sensing is considered. ROV operators find long duration tasks such as object search, cable tracking and object following very exhausting. An autonomous system to assist ROV operators will make the operations more reliable and efficient. Considering the above mentioned reasons this paper introduces visual sensors for target recognition and guidance of underwater vehicles (UVs).

Vision is a sensing modality rich with information of the short range environment and provides very high resolution compared with acoustic sensors ${ }^{1,5), 6,7,7), 11}$.

* Institute of Industrial Science, University of Tokyo.

Received 10th July 1996

Read at the Autumn meeting 14, 15th Nov. 1996
There are, however, certain features which are unique to the underwater environment yet undesirable for computer vision algorithms.

This paper discusses a filtering technique to minimize the effects due to the undesirable features of the underwater environment and proposes some techniques to use visual data for the guidance of UVs. The performance of the proposed system is demonstrated through direct application to typical underwater examples such as underwater cable tracking and object following based on the image captured by a commercially available Charge-Coupled Device (CCD) camera.

\section{Underwater Visual Sensing}

Visual sensing plays a main role in autonomous land systems as it is unobtrusive, accurate, and has a high bandwidth. There are, however, limitations due to both the environment and currently available technology. Due to the little or no ambient light underwater, the visibility is poor so that active light sources have to be used to illuminate the working environment resulting in non-uniform lighting. In case of AUVs, it is almost impossible to place large hardware systems in their small pressure hulls.

\subsection{Underwater optical properties}

An underwater image consists of several light sources reflected from the environment as shown in Fig. 1.

The linear superposition of the three reflected quantities shown in Fig. 1 are considered in the final image formed in the camera plane ;

$$
I_{t}(x, y)=I_{d}(x, y)+I_{b k}(x, y)+I_{b}(x, y)
$$

where,

$I_{t}=$ the total intensity,

$I_{d}=$ the direct component : the light reflected from the target, 


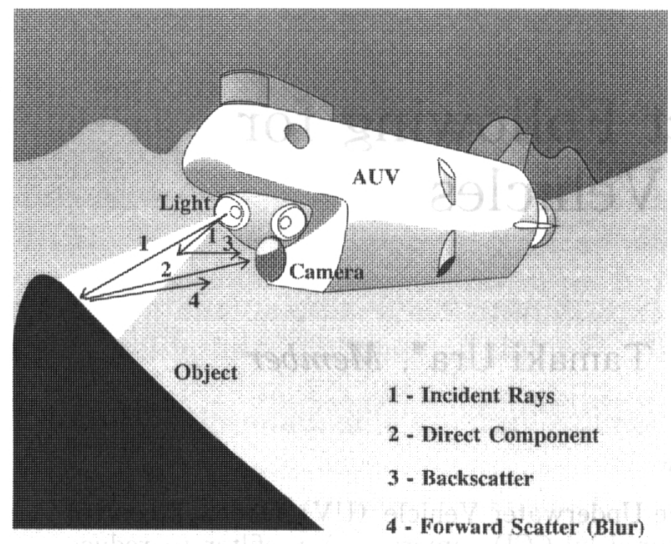

Fig. 1 Underwater optical properties

$I_{b k}=$ the backscatter component : light scattered by water and suspended particles with no illumination from the object, and

$I_{b}=$ the blur component (forward scatter) : the light reflected from the target can also be scattered on its way to the camera.

By considering each function ${ }^{4)}$;

$$
\begin{aligned}
& I_{d}(x, y)=\frac{I_{r}}{R^{\prime 2}}(x, y, z) K(\text { camera properties }) e^{-c R} \\
& I_{b}(x, y)=\frac{I_{d}}{e^{-c R}}\left(e^{-G R^{\prime}}-e^{-c R^{\prime}}\right)^{*} F^{-1}\left(e^{-B R^{\prime} f}\right) \\
& I_{b k}(x, y)=\sum_{n=1}^{z / \Delta z} I_{b p}(x, y, z)
\end{aligned}
$$

where

$I_{r}$ - reflectance intensity from the target

$I_{b p}$ - the intensity at the backscatter plane

$K$ - is a function depending on the camera used

$c$ - total attenuation coefficient

$R$-distance from the light source to the target

$R^{\prime}$ - distance from the target to the camera

$G$ - empirical constant related to the power in the scattered component

$B$-empirical constant related to the angular attenuation

$f$ - angular frequency in radians

$F^{-1}$ - inverse Fourier transform

$*$ - convolution

$Z$ - distance from the light source to the camera

$\Delta Z$ - increment in the $z$-direction

By considering the frequency response of the above optical properties as shown in Fig. 2, it is clear that underwater images have spatial attenuation of high frequencies (blur) and predominance of low frequencies (backscatter). A filter should be designed to filter out some of the low frequency components and to boost some high frequency components which were attenuated.

\section{2 Underwater image properties}

Unique features of the underwater images are mainly of three catogaries ;

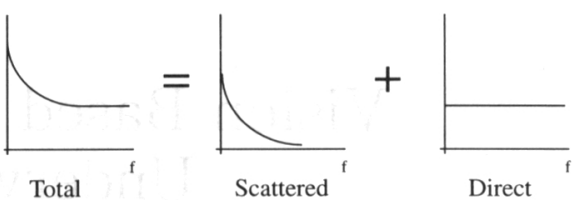

Fig. 2 Frequency response of the underwater optical properties

\section{(1) Non-uniform lighting}

Due to the light absorbed by water, far ranges will appear to be darker than the close ranges and highly directional active light sources make regions in the image to appear darker and brighter. This will result in a non-uniform lighting condition in the underwater image which is undesirable for most of the available computer vision algorithms.

(2) Suspended particles in water

The backscattering is mostly due to the randomly moving particles suspended in water. As a result, the image consists of many bright spots. In case of marine snow the image could be improved with the use of filtering techniques. But in case of high turbidity, quality of the image will be difficult to improve.

(3) Limited range

Due to absorption of light, the range of visibility underwater is limited

To enable underwater computer vision processing, a filtering technique should be introduced to reduce the above mentioned effects to some extent.

\section{Filtering of Underwater Images}

The scattering of light underwater introduces low frequency components to the image. The marine snow introduces not only backscattering but also high frequency components due to the fast moving particles. Therefore, it can be concluded that band pass filters will be the best to reduce the effects observed in underwater images.

In this paper, we propose to use the Laplacian of Gaussian (LoG) filter as the band limiting filter for underwater images ${ }^{3), 10)}$. The Laplacian portion will act as a high pass filter and the Gaussian as a low pass filter. Main advantage of this filter is that it can be implemented in real-time applications. The LoG mask in two dimensions is given in Eq. 3 .

$$
\nabla^{2} G(x, y)=\left[K\left(2-\frac{\left(x^{2}+y^{2}\right)}{\sigma^{2}}\right) e\left[-\frac{\left(x^{2}+y^{2}\right)}{2 \sigma^{2}}\right]\right]
$$

where $\sigma$ is the space constant of the Gaussian, $K$ is a scale factor and $(x, y)$ is the pixel position in the image. The size of the LoG operator, $s=8.5 \sigma$.

The characteristic curve of the LoG mask is shown in Fig. 3.

The filter response on an intensity variation in the image, in a particular direction is shown in Fig. 4. Note how the filter evaluates a zero at the local maxima. 


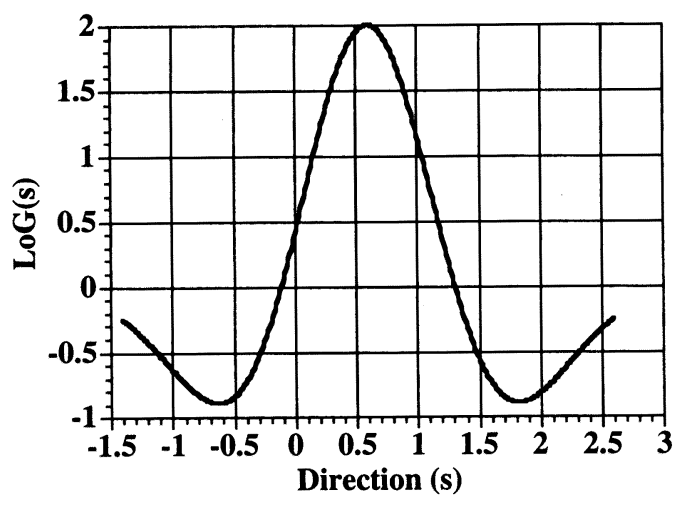

Fig. 3 The LoG mask

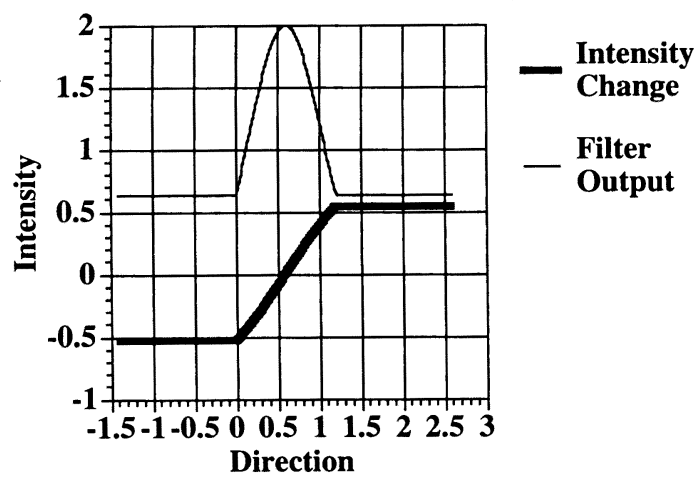

Fig. 4 The LoG response on an intensity variation

The zero crossings are locally more stable in the presence of noise than most other properties, thus making them more attractive for underwater images. Therefore, a signum function to extract the zero crossings from the LoG filtered image is proposed.

\section{Underwater Object Following}

Although the use of vision for dynamically servoing an autonomous system is a well studied area, the applications are either for land navigation or for a structured environment, such as a production cell ${ }^{8), 9)}$. UV navigation is more challenging due to the fact that it involves 6 degrees of freedom. The motion parameters of the object of interest which is to be followed can be derived with the pin-hole camera model shown in Fig. 5.

A point $\mathrm{P}$ projects into a point $\mathrm{p}$ in the image plane with image coordinates $(x, y)$ given by ;

$$
x=X_{s} / Z_{s} \text { and } y=Y_{s} / Z_{s}
$$

The perspective projection and the focal length is assumed to be unity. If the vehicle moves in a static environment with a translational velocity $T$ and with an angular velocity $R$ with respect to the vehicle frame, then the velocity of point $P$ can be derived as;

$$
\frac{d P}{d t}=-T-R \times P
$$

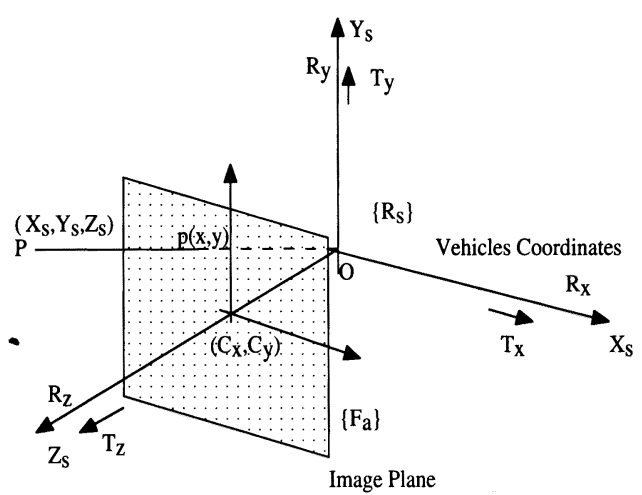

Fig. 5 Image and vehicle coordinate systems. Where $T_{x}$-Sway, $T_{y}$-Heave, $T_{z}$-Surge, $R_{x}$-Pitch, $R_{y}$ Yaw, $R_{z}$-Roll

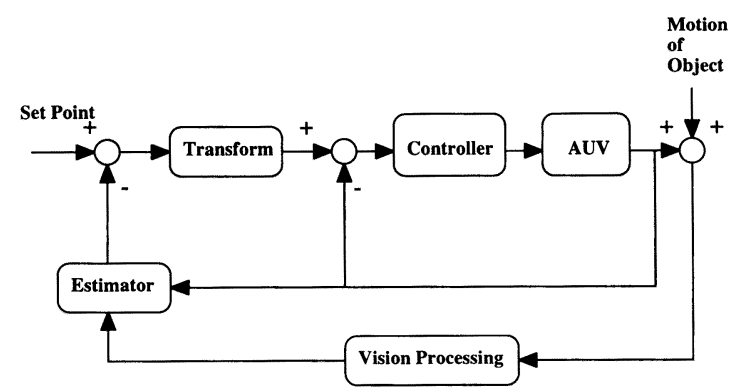

Fig. 6 The general schematic diagram of the vision based tracking system

By taking the time derivatives of the expressions for $x$ and $y$ and using (4) and (5), we obtain Eq. (6) which is used as the motion estimator in the proposed tracking algorithms.

$$
\begin{aligned}
& \dot{x}=\left[x \frac{T_{z}}{Z_{s}}-\frac{T_{x}}{Z_{s}}\right]+\left[x y R_{x}-(1+x)^{2} R_{y}+y R_{z}\right] \\
& \dot{y}=\left[y \frac{T_{z}}{Z_{s}}-\frac{T_{y}}{Z_{s}}\right]+\left[(1+y)^{2} R_{x}-x y R_{y}-x R_{z}\right]
\end{aligned}
$$

Two real-time vision based tracking algorithms are proposed for underwater vehicles: (1). Feature based object following ( 2 ). Template based object following. The general schematic diagram of the object following system is shown in Fig. 6 .

The set point is the target position desired in the image plane and the control commands are derived by comparing it with the current position of the target found by the vision processing algorithm. The estimator given in Eq. (6) will compensate the delay caused by the vision processing algorithm. The transform block in Fig. 6 will transform the error found in the $2 \mathrm{D}$ image plane to the $3 \mathrm{D}$ plane by using the pinhole camera model.

\section{1 Feature based object following}

This algorithm is capable of tracking objects consisting of well defined geometric features which will not change with change in pose. A typical example is 
underwater cable tracking. Navigation commands are derived using the position of the cable in the image. The position of the cable is found by extracting the linear features of the image.

As shown in Fig. 7, the equation of the line found by the vision processor will be compared with the equation of the line at the desired location.

\subsubsection{Line feature extraction}

Hough transformation technique is used to extract the line features from the image. In this technique a line, given in Eq. $(7)$, in the image plane $(x, y)$ is transformed into the parametric plane. Where the parameters of the line are shown in Fig. 8(a). A straight line in the image plane will correspond to a point in the parametric plane. During the transformation the number of pixels falling into each parametric coordinate will be counted as shown in Fig. 8( $\mathrm{b}$ ). The highest vote of pixels in the parametric plane will correspond to the longest line in the image plane. With the estimator given in Eq. (6), the where abouts of the line can be predicted and Hough transformation will be necessary only in that region to get the details of the line. Thus the extraction will be much faster enabling real-time operations and will avoid detecting false targets. As underwater environments will consist of only a very few well defined geometric shapes, Hough transformation technique is found to be suitable for underwater cable detection.

$$
r=x \cos (\theta)+y \sin (\theta)
$$

\section{2 Template based object following}

This technique is used in situations when a particular feature of the target cannot be defined. Fish following is a typical example of this nature. In this technique, a template will be derived using the image of the object to be tracked as shown in Fig. 9. This template will be correlated with the neighbourhood in the next frame with the correlation function $R(u, v)$ given in Eq. ( 8 ). The best correlation will correspond to the new location of the object. In order to cater for the changing shape and size of the interested object, the template is updated according to the variance and the mean at the best correlation.

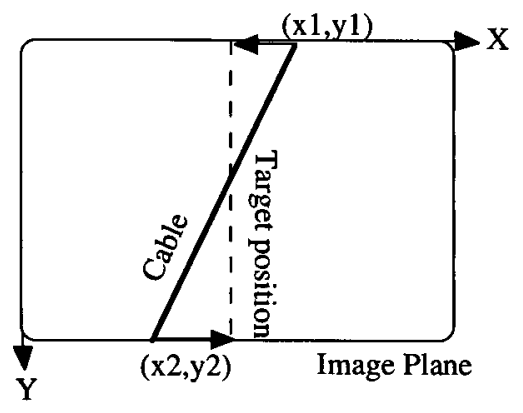

Fig. 7 Cable tracking example

$$
R(u, v)=\sum_{j=1}^{J} \sum_{k=1}^{K}[I(j, k) X O R I(j-u, k-v)]
$$

where, $u, v$ are the displacement of the target and $I(j$, $k$ ) is the intensity of the pixel at $(j, k)$. With the Signum of LoG (SoLoG) filter a binary image is obtained, therefore correlation can be easily calculated with the binary exclusive OR (XOR) operation.

\section{Experimental Results}

\section{1 Performance of the SoLoG filter}

The performance of the SoLoG filter was tested on real images captured at a depth of $1000 \mathrm{~m}$ with a CCD

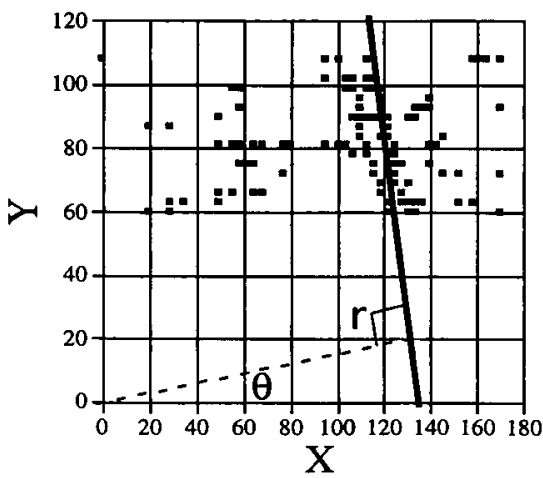

(a) Image plane

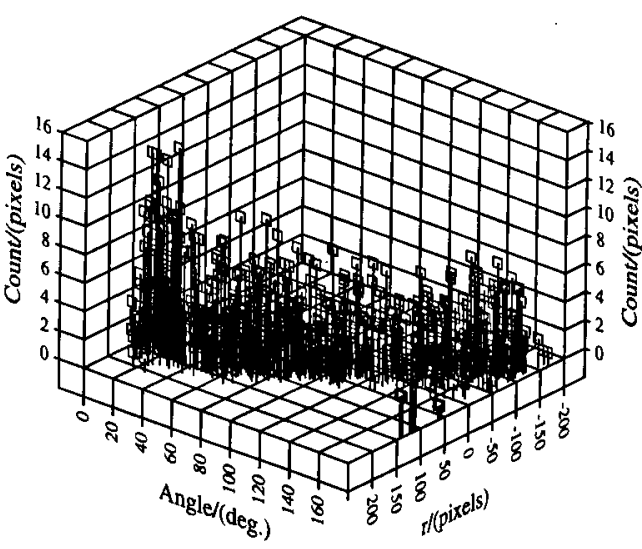

(b) Parametric space

Fig. 8 Hough transformation

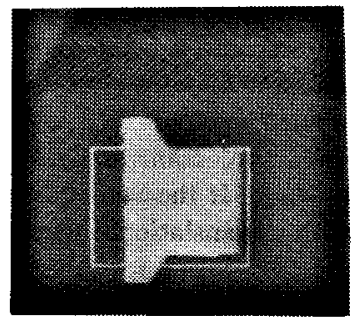

Fig. 9 The tracking template 
camera mounted on the Dolphin $3 \mathrm{~K}$ ROV. From the Figs. 10 (a) and 11(a) it can be seen how the underwater images are corrupted with the features mentioned in section 2. In Fig. 10 (a), there is an eel (left center), but due to the predominance of backscatter the details of the fish can hardly be seen. As it can be seen, the highly directional light source of the ROV had made the light condition to be non-uniform as well. Figure 10 (b) shows how the filter had reduced the noises making the details of the target visible. Similar effects are shown in Fig. 11(b). Note the marine snow effect in Fig. 11 ( a ). Figure 11 (c) shows the binary image as a result of the signum operation on the LoG filtered image. These results show the strength of the LoG filter in minimizing the special features discussed in section 2.

\section{2 Feature based object following}

The experiments were carried out by placing a yellow cable on the bottom of lake Biwako and on the bottom of the experimental pool at the University of Tokyo. The algorithm discussed in section 4.1 was implemented in the hardware of the test-bed vehicle "The Twin-Burger". It is a INMOS B 429 transputer based hardware system which can be placed easily in the pressure hull of an underwater vehicle. The processing time of the algorithm on this hardware is 3 decisions/sec..

Figure 12 shows how Hough transformation technique was successful in determining the position of the cable from the image of lake Biwako. The dark dots in Fig. 12(b) represent the detected line. The performance of the algorithm was tested in a situation when there are many linear features in the environment as shown in Fig. 13. With the use of the estimator given in Eq. ( 6 ), the algorithm performed very well in following the cable. The white dots in Fig. 13(b) show the position of the line detected by the algorithm.

\section{3 Template based object following}

The object in the template shown in Fig. 9 was moved in a known trajectory. The Fig. 14 shows the movement of the object in a circular trajectory. From the trajectory of the coordinates of the template centroid as shown in Fig. 15, it can be concluded that template followed the object successfully. The algorithm was implemented in the Max Video image processing board and the speed of following the target was at 4 frames/ sec.. This experiment describes how an object could be

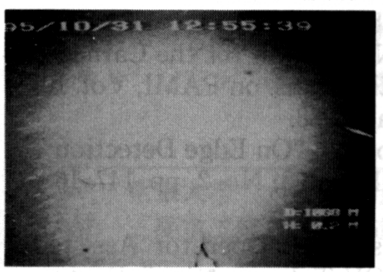

(a) Original

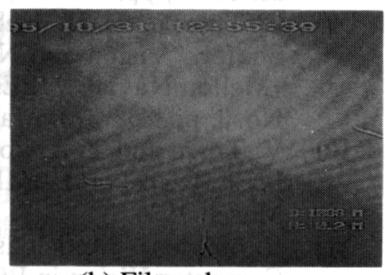

(b) Filtered
Fig. 10 LoG filter example 1

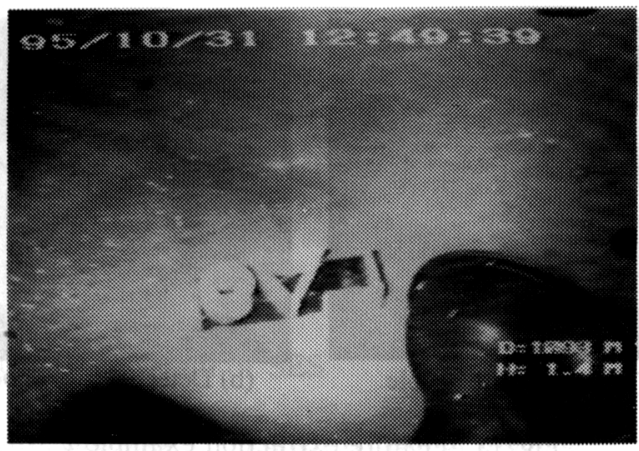

(a) Original image

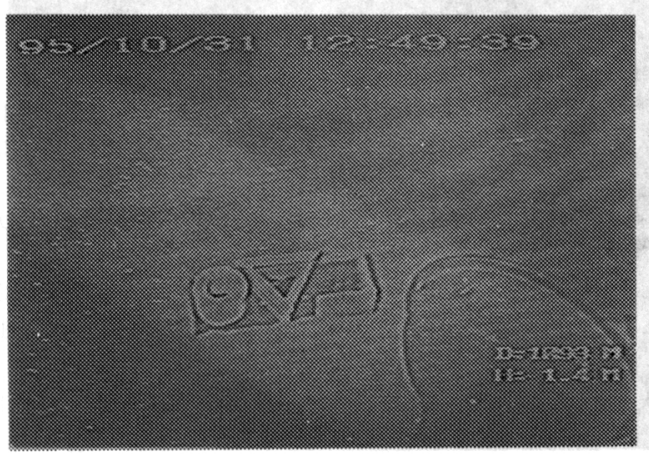

(b) LoG image

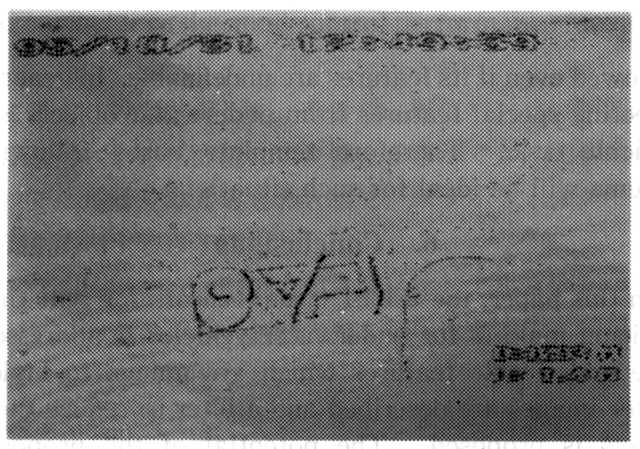

(c) SoLoG image

Fig. 11 LoG filter example 2

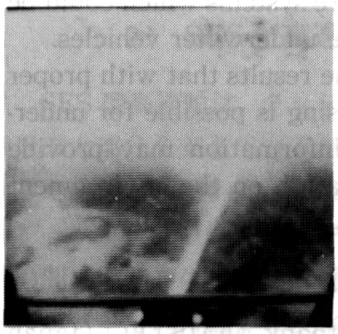

(a) Image of the cable

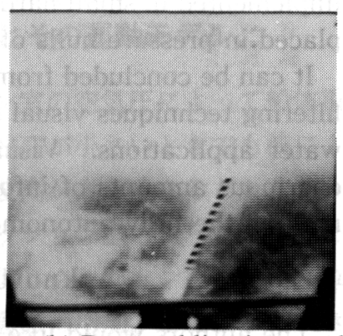

(b) Detected line imposed
Fig. 12 Feature extraction example 1 


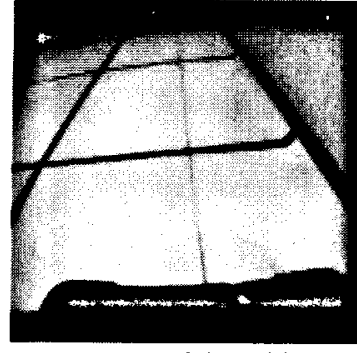

(a) Image of the cable

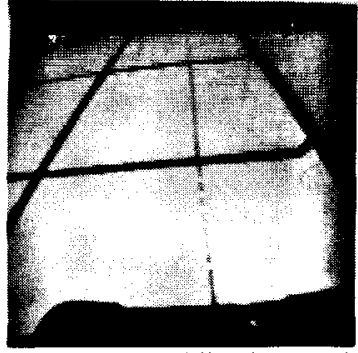

(b) Detected line imposed

Fig. 13 Feature extraction example 2

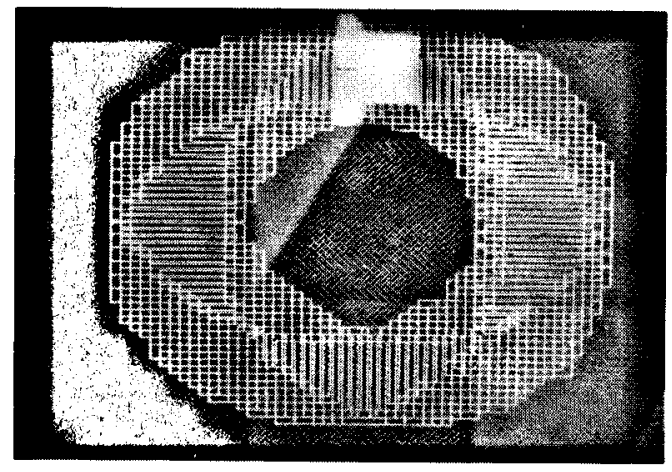

Fig. 14 Template based object following

followed even if its features are undefinable. In general, selecting special features from underwater objects is a difficult task. Therefore template based following scheme will be ideal for such situations.

\section{Conclusions}

In this paper, the use of visual sensors for real-time decision making for underwater vehicles is addressed. The undesirable features which are unique to underwater images are described and a filter to reduce these effects is proposed. The potential of the proposed approaches have been demonstrated by experimental results from applications such as underwater cable tracking and underwater object following. The algorithms were developed in such a way that they can be implemented in small hardware systems which could be placed in pressure hulls of the underwater vehicles.

It can be concluded from the results that with proper filtering techniques visual sensing is possible for under. water applications. Visual information may provide enormous amounts of information on the environment making the fully autonomous system a reality.

\section{Acknowledgement}

The authors would like to thank JAMSTEC (Japan Marine Science and Technology Center) and the Biwa-

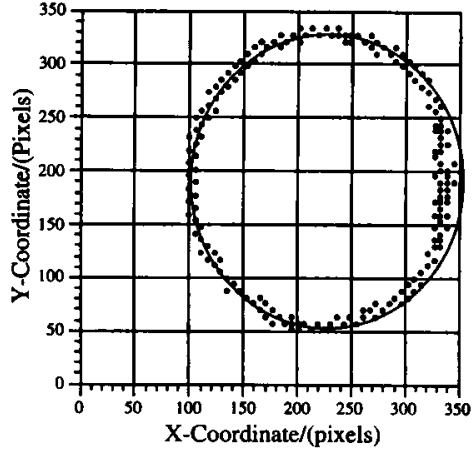

Template Trajectory Trajectory of the obje

Fig. 15 Trajectory of the centroid of the template

ko Research Institute for providing underwater images.

\section{References}

1) B. A. A. P Balasuriya and Tamaki Ura, "Vision Based Tracking for Unmanned Underwater Vehicles", Proc. of SICE '96, July 1996, pp. 1077-1081.

2) R. Blidberg, "Autonomous underwater vehicles, Current activities and research opportunities", Int. Rob. Aut. Sys., vol. 7., No. 2-3, pp. 139-150, August 1991.

3) A. Huertas and G. Medioni, "Detection of Intensity Changes with Subpixel Accuracy Using La. placian-Gaussian Masks", IEEE Trans. on PAMI, Vol. 8, No. 5, pp. 651-664, September 1986.

4) Jules S. Jaffe, "Computer Modeling and the Design of Optimal Underwater Imaging Systems", IEEE J. of Oceanic Eng., Vol. 15, No.2, April 1990, pp. 101-111.

5) R. L. Marks, Michael J. Lee and Stephen M. Rock, "Automatic Object Tracking for an Unmanned Underwater Vehicle using Real-Time Filtering and Correlation", Proc. of IEEE Sys., Man and Cybernetics, pp. 337-342, France, October 1993.

6) S. Matsumoto and Y. Ito, "Vision-Based Tracking of Submarine Cables", Proc. of 13th Ocean Eng. Sym., pp. 365-370, July 1995 (in Japanese).

7) S. Negahdaripour, A. Shokrollahi, J. Fox and S. Arora, "Improved Methods for Undersea Optical Stationkeeping", Proc. of IEEE Intl. conf. on Robotics and Automation, California, pp. 27522758, April 1991.

8) N. P. Papanikolopoulos, P. K. Khosla, "Adaptive Robotic Visual Tracking: Theory and Experiments", IEEE Trans. on Automatic Control, Vol. 38, No. 3, pp. 429-444, March 1993.

9) C. Thorpe, M. H. Hebert, T. Kanade and S. A. Shafer, "Vision and Navigation for the Carnegie -Mellon Navlab", IEEE Trans. on PAMI, Vol. 10, No. 3, pp. 362-373, May 1988.

10) V. Torre and T. A. Poggio, "On Edge Detection", IEEE Trans. on PAMI, Vol. 8, No. 2, pp. 147-163, March 1986.

11) R. Volden, "Modelbased and Operator Assisted Computer Vision in ROV Teleoperation", Doctaral Thesis, NTH, 1995. 